\title{
Stability and Hopf Bifurcation Analysis of Coupled Optoelectronic Feedback Loops
}

\author{
Gang $\mathrm{Zhu}^{1,2}$ and Junjie Wei ${ }^{1}$ \\ ${ }^{1}$ Department of Mathematics, Harbin Institute of Technology, Harbin, Heilongjiang 150001, China \\ ${ }^{2}$ School of Education Science, Harbin University, Harbin, Heilongjiang 150086, China \\ Correspondence should be addressed to Junjie Wei; weijj@hit.edu.cn
}

Received 7 January 2013; Accepted 28 January 2013

Academic Editor: Patricia J. Y. Wong

Copyright (c) 2013 G. Zhu and J. Wei. This is an open access article distributed under the Creative Commons Attribution License, which permits unrestricted use, distribution, and reproduction in any medium, provided the original work is properly cited.

\begin{abstract}
The dynamics of a coupled optoelectronic feedback loops are investigated. Depending on the coupling parameters and the feedback strength, the system exhibits synchronized asymptotically stable equilibrium and Hopf bifurcation. Employing the center manifold theorem and normal form method introduced by Hassard et al. (1981), we give an algorithm for determining the Hopf bifurcation properties.
\end{abstract}

\section{Introduction}

In recent research [1-5], it is found that even if several individual systems behave chaotically, in the case where the systems are identical, by proper coupling, the systems can be made to evolve toward a situation of exact isochronal synchronism. Synchronization phenomena are common in coupled semiconductor systems, and they are important examples of oscillators in general, and many works are concerned with coupled semiconductor systems [6-15].

We consider a feedback loop comprises a semiconductor laser that serves as the optical source, a Mach-Zehnder electrooptic modulator, a photoreceiver, an electronic filter, and an amplifier. The dynamics of the feedback loop can be modeled by the delay differential equations $[14,15]$ :

$$
\begin{aligned}
\frac{\mathrm{d} x_{1}(t)}{\mathrm{d} t}= & -\left(\gamma_{1}+\gamma_{2}\right) x_{1}(t)-\gamma_{2} y_{1}(t) \\
& -\beta \gamma_{2} \cos ^{2}\left[x_{1}(t-\tau)+\varphi_{0}\right], \\
\frac{\mathrm{d} y_{1}(t)}{\mathrm{d} t}= & \gamma_{1} x_{1}(t) .
\end{aligned}
$$

Here, $x_{1}(t)$ is the normalized voltage signal applied to the electrooptic modulator, $\tau$ is the feedback time delay, $\gamma_{1}$ and $\gamma_{2}$ are the filter low-pass and high-pass corner frequencies, $\beta$ is the dimensionless feedback strength, they are all positive constants, and $\varphi_{0}$ is the bias point of the modulator.

Depending on the value of the feedback strength $\beta$ and delay $\tau$, the loop, which is modeled by system (1), is capable of producing dynamics ranging from periodic oscillations to high-dimensional chaos $[1,14,15]$.

We couple two nominally identical optoelectronic feedback loops unidirectionally, that is, the transmitter affects the dynamics of the receiver but not vice versa. Thus, the equations of motion describing the coupled system are given by (1) for the transmitter and

$$
\begin{aligned}
\frac{\mathrm{d} x_{2}(t)}{\mathrm{d} t}= & -\left(\gamma_{1}+\gamma_{2}\right) x_{2}(t)-\gamma_{2} y_{2}(t) \\
& -\beta \gamma_{2} \cos ^{2}\left[k x_{1}(t-\tau)+(1-k) x_{2}(t-\tau)+\varphi_{0}\right], \\
\frac{\mathrm{d} y_{2}(t)}{\mathrm{d} t}= & \gamma_{1} x_{2}(t),
\end{aligned}
$$

for the receiver. In (2), $k>0$ denotes the coupling strength. We will find that with the variety of $k$, the dynamical behavior of the coupled system can be different, while the feedback strength $\beta$ keeps the same value.

The paper is organized as follows. In Section 2, using the method presented in [16], we study the stability, and 
the local Hopf bifurcation of the equilibrium of the coupled system (1) and (2) by analyzing the distribution of the roots of the associated characteristic equation. In Section 3, we use the normal form method and the center manifold theory introduced by Hassard et al. [17] to analyze the direction, stability and the period of the bifurcating periodic solutions at critical values of $\beta$. In Section 4 , some numerical simulations are carried out to illustrate the results obtained from the analysis. In Section 5, we come to some conclusion about the effect caused by the variety of parameters.

\section{Stability Analysis}

In this section, we consider the linear stability of the nonlinear coupled system

$$
\begin{aligned}
\frac{\mathrm{d} x_{1}(t)}{\mathrm{d} t}= & -\left(\gamma_{1}+\gamma_{2}\right) x_{1}(t)-\gamma_{2} y_{1}(t) \\
& -\beta \gamma_{2} \cos ^{2}\left[x_{1}(t-\tau)+\varphi_{0}\right], \\
\frac{\mathrm{d} y_{1}(t)}{\mathrm{d} t}= & \gamma_{1} x_{1}(t), \\
\frac{\mathrm{d} x_{2}(t)}{\mathrm{d} t}= & -\left(\gamma_{1}+\gamma_{2}\right) x_{2}(t)-\gamma_{2} y_{2}(t) \\
& -\beta \gamma_{2} \cos ^{2}\left[k x_{1}(t-\tau)+(1-k) x_{2}(t-\tau)+\varphi_{0}\right], \\
\frac{\mathrm{d} y_{2}(t)}{\mathrm{d} t}= & \gamma_{1} x_{2}(t) .
\end{aligned}
$$

It is easy to see that $E\left(0,-\beta \cos ^{2} \varphi_{0}, 0,-\beta \cos ^{2} \varphi_{0}\right)$ is the only equilibrium of system (3). Linearizing system (3) around $E$ and denote $\delta=\sin 2 \varphi_{0}$, we get the linearization system

$$
\begin{aligned}
\frac{\mathrm{d} x_{1}(t)}{\mathrm{d} t}= & -\left(\gamma_{1}+\gamma_{2}\right) x_{1}(t)-\gamma_{2} y_{1}(t)+\beta \delta \gamma_{2} x_{1}(t-\tau), \\
\frac{\mathrm{d} y_{1}(t)}{\mathrm{d} t}= & \gamma_{1} x_{1}(t), \\
\frac{\mathrm{d} x_{2}(t)}{\mathrm{d} t}= & -\left(\gamma_{1}+\gamma_{2}\right) x_{2}(t)-\gamma_{2} y_{2}(t) \\
& +k \beta \delta \gamma_{2} x_{1}(t-\tau)+(1-k) \beta \delta \gamma_{2} x_{2}(t-\tau), \\
\frac{\mathrm{d} y_{2}(t)}{\mathrm{d} t}= & \gamma_{1} x_{2}(t),
\end{aligned}
$$

and the characteristic equation of system (4)

$$
\begin{aligned}
& {\left[\lambda^{2}+\left(\gamma_{1}+\gamma_{2}\right) \lambda+\gamma_{1} \gamma_{2}-(1-k) \beta \delta \gamma_{2} \lambda e^{-\lambda \tau}\right]} \\
& \quad \times\left[\lambda^{2}+\left(\gamma_{1}+\gamma_{2}\right) \lambda+\gamma_{1} \gamma_{2}-\beta \delta \gamma_{2} \lambda e^{-\lambda \tau}\right]=0
\end{aligned}
$$

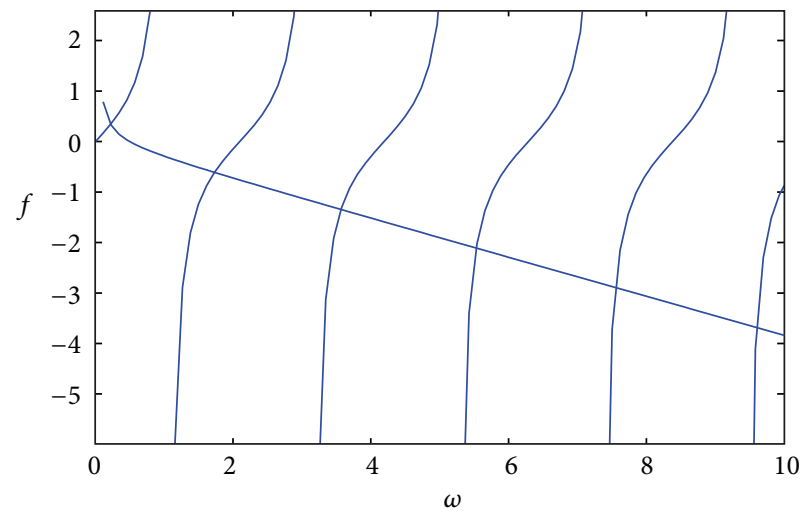

FIGURE 1: The points of intersection of $f_{1}=\tan \omega \tau$ and $f_{2}=\left(-\omega^{2}+\right.$ $\left.\gamma_{1} \gamma_{2}\right) / \omega\left(\gamma_{1}+\gamma_{2}\right)$, when $\gamma_{1}=0.1, \gamma_{2}=2.5, \tau=1.5$.

which is equivalent to

$$
\begin{gathered}
\lambda^{2}+\left(\gamma_{1}+\gamma_{2}\right) \lambda+\gamma_{1} \gamma_{2}-\beta \delta \gamma_{2} \lambda e^{-\lambda \tau}=0, \\
\lambda^{2}+\left(\gamma_{1}+\gamma_{2}\right) \lambda+\gamma_{1} \gamma_{2}-(1-k) \beta \delta \gamma_{2} \lambda e^{-\lambda \tau}=0 .
\end{gathered}
$$

Notice that when $\beta=0$, (5) becomes

$$
\left[\lambda^{2}+\left(\gamma_{1}+\gamma_{2}\right) \lambda+\gamma_{1} \gamma_{2}\right]^{2}=0
$$

whose roots are

$$
\lambda_{1,2}=-\gamma_{1}, \quad \lambda_{3,4}=-\gamma_{2} .
$$

So, we have the following lemma.

Lemma 1. The equilibrium $E_{0}\left(0,-\beta \cos ^{2} \varphi_{0}, 0,-\beta \cos ^{2} \varphi_{0}\right)$ is asymptotically stable when $\beta=0$.

Next, we regard $\beta$ as the bifurcation parameter to investigate the distribution of roots of (6) and (7).

Let $\lambda=i \omega(\omega>0)$ be a root of (6) and substituting $\lambda=i \omega$ into (6), separating the real and imaginary parts yields

$$
\begin{aligned}
& -\omega^{2}+\gamma_{1} \gamma_{2}=\beta \delta \gamma_{2} \omega \sin \omega \tau, \\
& \omega\left(\gamma_{1}+\gamma_{2}\right)=\beta \delta \gamma_{2} \omega \cos \omega \tau .
\end{aligned}
$$

Then, we can get

$$
\tan \omega \tau=\frac{-\omega^{2}+\gamma_{1} \gamma_{2}}{\omega\left(\gamma_{1}+\gamma_{2}\right)}
$$

Hence, (11) has a sequence of roots $\left\{\omega_{j}\right\}_{j \geq 0}$ (see Figure 1), and

$$
\omega_{j} \in \begin{cases}\left(\frac{2 j \pi}{\tau}, \frac{2 j \pi+\pi / 2}{\tau}\right), & \omega_{j}^{2}<\gamma_{1} \gamma_{2}, \\ \left(\frac{2 j \pi+3 \pi / 2}{\tau}, \frac{2(j+1) \pi}{\tau}\right), & \omega_{j}^{2}>\gamma_{1} \gamma_{2}, \\ j=0,1,2, \ldots\end{cases}
$$


Define

$$
\beta_{j}=\frac{\gamma_{1}+\gamma_{2}}{\delta \gamma_{2} \cos \omega_{j} \tau}
$$

Then, $\left(\omega_{j}, \beta_{j}\right)$ is the solution of $(10)$.

From (10), we know that

$$
\beta^{2}=\frac{1}{\delta^{2} \gamma_{2}^{2}}\left[\left(\frac{\gamma_{1} \gamma_{2}}{\omega}-\omega\right)^{2}+\left(\gamma_{1}+\gamma_{2}\right)^{2}\right],
$$

which gives that

$$
\frac{\mathrm{d} \beta}{\mathrm{d} \omega}=\frac{1}{\beta \delta^{2} \gamma_{2}^{2} \omega^{3}}\left(\omega^{2}+\gamma_{1} \gamma_{2}\right)\left(\omega^{2}-\gamma_{1} \gamma_{2}\right) .
$$

From Figure 1, we know that $\omega_{j} \rightarrow \infty$ when $j \rightarrow \infty$, which means that $\omega_{j}^{2}>\gamma_{1} \gamma_{2}$; furthermore, $\beta$ is increasing with respect to $\omega$, when $j$ is sufficiently big.

Reorder the set $\left\{\beta_{j}\right\}$ such that $\beta_{0}=\min \left\{\beta_{j}\right\}$ and $\omega_{j}$ is correspondent of $\beta_{j}(j=0,1,2, \ldots)$. Then, we have the following lemma.

Lemma 2. There exists a sequence values of $\beta$ denoted by

$$
0<\beta_{0}<\beta_{1}<\cdots
$$

such that (6) has a pair of imaginary roots $\pm i \omega_{j}$ when $\beta=$ $\beta_{j}(j=0,1,2, \ldots)$, where $\beta_{j}$ is defined by (13), and $\omega_{j}$ is the root of (11).

Let

$$
\lambda(\tau)=\alpha(\beta)+i \omega(\beta)
$$

be the root of (6) satisfying $\alpha\left(\beta_{j}\right)=0$ and $\omega\left(\beta_{j}\right)=\omega_{j}$. We have the following conclusion.

Lemma 3. $\alpha^{\prime}\left(\beta_{j}\right)>0$.

Proof. Substituting $\lambda(\beta)$ into (6) and taking the derivative with respect to $\beta$, it follows that

$$
\begin{aligned}
2 \lambda \frac{\mathrm{d} \lambda}{\mathrm{d} \beta} & +\left(\gamma_{1}+\gamma_{2}\right) \frac{\mathrm{d} \lambda}{\mathrm{d} \beta}-\delta \gamma_{2} \lambda e^{-\lambda \tau}-\beta \delta \gamma_{2} e^{-\lambda \tau} \frac{\mathrm{d} \lambda}{\mathrm{d} \beta} \\
& +\tau \beta \delta \gamma_{2} \lambda e^{-\lambda \tau} \frac{\mathrm{d} \lambda}{\mathrm{d} \beta}=0 .
\end{aligned}
$$

Therefore, noting that $\beta \delta \gamma_{2} \lambda e^{-\lambda \tau}=\lambda^{2}+\left(\gamma_{1}+\gamma_{2}\right) \lambda+\gamma_{1} \gamma_{2}$, we have

$$
\frac{\mathrm{d} \lambda}{\mathrm{d} \beta}=\frac{1}{\beta} \frac{\lambda^{3}+\left(\gamma_{1}+\gamma_{2}\right) \lambda^{2}+\gamma_{1} \gamma_{2} \lambda}{\lambda^{2}-\gamma_{1} \gamma_{2}+\tau\left[\lambda^{3}+\left(\gamma_{1}+\gamma_{2}\right) \lambda^{2}+\gamma_{1} \gamma_{2} \lambda\right]},
$$

and by a straight computation, we get

$$
\begin{gathered}
\alpha^{\prime}\left(\beta_{j}\right)=\frac{\omega_{j}^{2}}{\beta_{j} \Delta}\left[\tau \omega_{j}^{2}\left(\gamma_{1}^{2}+\gamma_{2}^{2}\right)+\left(\omega_{j}^{2}+\gamma_{1} \gamma_{2}\right)\left(\gamma_{1}+\gamma_{2}\right)\right. \\
\left.+\tau \omega_{j}^{4}+\tau \gamma_{1}^{2} \gamma_{2}^{2}\right]>0
\end{gathered}
$$

where

$$
\begin{aligned}
\Delta= & {\left[\left(\omega_{j}^{2}+\gamma_{1} \gamma_{2}\right)+\tau \omega_{j}^{2}\left(\gamma_{1}+\gamma_{2}\right)\right]^{2} } \\
& +\left[\tau \omega_{j}\left(\omega_{j}^{2}-\gamma_{1} \gamma_{2}\right)\right]^{2}
\end{aligned}
$$

As to (7), it can be easily found that $-\gamma_{1},-\gamma_{2}$ are two negative roots when $k=1$, so, next, we only focus on (7) with $k \neq 1$.

Let $\lambda=i \omega(\omega)>0$ be a root of (7). Using the same method above, we get

$$
\begin{gathered}
-\omega^{2}+\gamma_{1} \gamma_{2}=(1-k) \beta \delta \gamma_{2} \omega \sin \omega \tau, \\
\left(\gamma_{1}+\gamma_{2}\right) \omega=(1-k) \beta \delta \gamma_{2} \omega \cos \omega \tau, \\
\tan \omega \tau=\frac{-\omega^{2}+\gamma_{1} \gamma_{2}}{\omega\left(\gamma_{1}+\gamma_{2}\right)} .
\end{gathered}
$$

Then, when $0<k<1$, (23) has a sequence of roots $\left\{\omega_{j}\right\}_{j \geq 0}$, which are the same as those of (11).

When $k>1$, (23) has a sequence of roots $\left\{\omega_{j}\right\}_{j \geq 0}$, and

$$
\omega_{j} \in \begin{cases}\left(\frac{(2 j+1) \pi}{\tau}, \frac{(2 j+1) \pi+\pi / 2}{\tau}\right), & \omega_{j}^{2}<\gamma_{1} \gamma_{2}, \\ \left(\frac{2 j \pi+\pi / 2}{\tau}, \frac{(2 j+1) \pi}{\tau}\right), & \omega_{j}^{2}>\gamma_{1} \gamma_{2}, \\ j=0,1,2, \ldots\end{cases}
$$

Define

$$
\bar{\beta}_{j}=\frac{\gamma_{1}+\gamma_{2}}{(1-k) \delta \gamma_{2} \cos \omega \tau} .
$$

Then, $\left(\omega_{j}, \bar{\beta}_{j}\right)$ is the solution of (22).

Repeat the previous process, we have

$$
\frac{\mathrm{d} \bar{\beta}}{\mathrm{d} \omega}=\frac{1}{(1-k)^{2} \beta \delta^{2} \gamma_{2}^{2} \omega^{3}}\left(\omega^{2}+\gamma_{1} \gamma_{2}\right)\left(\omega^{2}-\gamma_{1} \gamma_{2}\right) \text {. }
$$

Reorder the set $\left\{\bar{\beta}_{j}\right\}$ such that $\bar{\beta}_{0}=\min \left\{\bar{\beta}_{j}\right\}$ and $\omega_{j}$ is correspondent of $\bar{\beta}_{j}(j=0,1,2, \ldots)$.

Lemma 4. There exists a sequence values of $\bar{\beta}$ denoted by

$$
0<\bar{\beta}_{0}<\bar{\beta}_{1}<\cdots
$$

such that (7) has a pair of imaginary roots $\pm i \omega_{j}$ when $\bar{\beta}=$ $\bar{\beta}_{j}(j=0,1,2, \ldots)$, where $\bar{\beta}_{j}$ is defined by (25), and $\omega_{j}$ is the root of (23).

Let

$$
\lambda(\tau)=\alpha(\bar{\beta})+\mathrm{i} \omega(\bar{\beta})
$$

be the root of (7) satisfying $\alpha\left(\bar{\beta}_{j}\right)=0, \omega\left(\bar{\beta}_{j}\right)=\omega_{j}$. Then, similar to the proof of Lemma 3 , we have the following conclusion. 
Lemma 5. $\alpha^{\prime}\left(\bar{\beta}_{j}\right)>0$.

Compare $\beta_{j}, \bar{\beta}_{j}$ and reorder the set $\left\{\beta_{j}\right\}$ and $\left\{\bar{\beta}_{j}\right\}$ and remove the "-" of $\bar{\beta}_{j}$, such that

$$
0<\beta_{0}<\beta_{1}<\cdots,
$$

then from previous lemmas and the Hopf bifurcation theorem for functional differential equations [18], we have the following results on stability and bifurcation to system (3).

Theorem 6. For system (3), the equilibrium $E$ is asymptotically stable when $\beta \in\left[0, \beta_{0}\right)$ and unstable when $\beta \in\left(\beta_{0},+\infty\right)$; system (3) undergoes a Hopf bifurcation at $E$ when $\beta=\beta$, $j=0,1,2, \ldots$, where $\beta_{j}$ are defined by (13) or (25).

\section{The Direction and Stability of the Hopf Bifurcation}

In Section 2 we obtained some conditions under which system (3) undergoes the Hopf bifurcation at some critical values of $\beta$. In this section, we study the direction, stability, and the period of the bifurcating periodic solutions. The method we used is based on the normal form method and the center manifold theory introduced by Hassard et al. [17].

Move $E\left(0,-\beta \cos ^{2} \varphi_{0}, 0,-\beta \cos ^{2} \varphi_{0}\right)$ to the origin $O(0,0$, $0,0)$ and denote $\delta=\sin 2 \varphi_{0}, \rho=\cos 2 \varphi_{0}$, then system (3) can be written as the form

$$
\begin{aligned}
\frac{\mathrm{d} x_{1}(t)}{\mathrm{d} t}= & -\left(\gamma_{1}+\gamma_{2}\right) x_{1}(t)-\gamma_{2} y_{1}(t)+\beta \delta \gamma_{2} x_{1}(t-\tau) \\
& +\beta \gamma_{2} \rho \varphi_{0} x_{1}^{2}(t-\tau)-\frac{2}{3} \beta \delta \gamma_{2} x_{1}^{3}(t-\tau)+O(4), \\
\frac{\mathrm{d} y_{1}(t)}{\mathrm{d} t}=\gamma_{1} x_{1}(t), & \\
\frac{\mathrm{d} x_{2}(t)}{\mathrm{d} t}=-\left(\gamma_{1}+\gamma_{2}\right) x_{2}(t)-\gamma_{2} y_{2}(t) & \\
& +\beta \gamma_{2}\left[k \delta x_{1}(t-\tau)+(1-k) \delta x_{2}(t-\tau)\right. \\
& +k^{2} \rho x_{1}^{2}(t-\tau) \\
& +2 k(1-k) \rho x_{1}(t-\tau) x_{2}(t-\tau) \\
& +(1-k)^{2} \rho x_{2}^{2}(t-\tau)-\frac{2}{3} k^{3} \delta x_{1}^{3}(t-\tau) \\
& -2 k^{2}(1-k) \delta x_{1}^{2}(t-\tau) x_{2}(t-\tau) \\
& -2 k(1-k)^{2} \delta x_{1}(t-\tau) x_{2}^{2}(t-\tau) \\
& \left.-\frac{2}{3}(1-k)^{3} \delta x_{2}^{3}(t-\tau)\right]+O(4), \\
\frac{\mathrm{d} y_{2}(t)}{\mathrm{d} t}=\gamma_{1} x_{2}(t) . &
\end{aligned}
$$

Clearly, the phase space is $\mathscr{C}=\mathscr{C}\left([-\tau, 0], \mathbb{R}^{4}\right)$. For convenience, let

$$
\beta^{*} \in\left\{\beta_{j}\right\} \cup\left\{\bar{\beta}_{j}\right\}
$$

and $\beta=\beta^{*}+\mu, \mu \in \mathbb{R}$. From the analysis above we know that $\mu=0$ is the Hopf bifurcation value for system(30). Let $i \omega^{*}$ be the root of the characteristic equation associate with the linearization of system (30) when $\beta=\beta^{*}$. For $\phi=\left(\phi_{1}, \phi_{2}, \phi_{3}, \phi_{4}\right) \in \mathscr{C}$, let

$$
L_{\mu}(\phi)=B \phi(0)+C \phi(-\tau),
$$

where

$$
\begin{aligned}
B= & \left(\begin{array}{cccc}
-\left(\gamma_{1}+\gamma_{2}\right) & -\gamma_{2} & 0 & 0 \\
\gamma_{1} & 0 & 0 & 0 \\
0 & 0 & -\left(\gamma_{1}+\gamma_{2}\right) & -\gamma_{2} \\
0 & 0 & \gamma_{1} & 0
\end{array}\right), \\
C & =\left(\begin{array}{cccc}
\beta \delta \gamma_{2} & 0 & 0 & 0 \\
0 & 0 & 0 & 0 \\
k \beta \delta \gamma_{2} & 0 & (1-k) \beta \delta \gamma_{2} & 0 \\
0 & 0 & 0 & 0
\end{array}\right) .
\end{aligned}
$$

By the Rieze representation theorem, there exists a $4 \times 4$ matrix, $\eta(\theta, \mu)(-\tau \leq \theta \leq 0)$, whose elements are of bounded variation functions such that

$$
L_{\mu}(\phi)=\int_{-\tau}^{0} \mathrm{~d} \eta(\theta, \mu) \phi(\theta), \quad \phi \in \mathscr{C} .
$$

In fact, we can choose

$$
\eta(\theta, \mu)= \begin{cases}B, & \theta=0, \\ 0, & \theta \in(-\tau, 0) \\ -C, & \theta=-\tau .\end{cases}
$$

Then, (30) is satisfied.

For $\phi \in \mathscr{C}$, define the operator $A(\mu)$ as

$$
A(\mu) \phi(\theta)= \begin{cases}\frac{\mathrm{d} \phi(\theta)}{\mathrm{d} \theta}, & \theta \in[-\tau, 0), \\ \int_{-\tau}^{0} \mathrm{~d} \eta(t, \mu) \phi(t), & \theta=0,\end{cases}
$$

and $R(\mu) \phi$ as

$$
R(\mu) \phi(\theta)= \begin{cases}0, & \theta \in[-\tau, 0), \\ f(\mu, \phi), & \theta=0,\end{cases}
$$

where 


$$
f(\mu, \phi)=\beta^{*} \gamma_{2}\left(\begin{array}{l}
\rho \phi_{1}^{2}(-\tau)-\frac{2}{3} \delta \phi_{1}^{3}(-\tau)+O(4) \\
0 \\
k^{2} \rho \phi_{1}^{2}(-\tau)+k(1-k) \rho \phi_{1}(-\tau) \phi_{3}(-\tau) \\
+(1-k)^{2} \rho \phi_{3}^{2}(-\tau)-\frac{2}{3} k^{3} \delta \phi_{1}^{3}(-\tau)-2 k^{2}(1-k) \delta \phi_{1}^{2}(-\tau) \phi_{3}(t-\tau) \\
-2 k(1-k)^{2} \delta \phi_{1}(-\tau) \phi_{3}^{2}(-\tau)-\frac{2}{3}(1-k)^{3} \delta \phi_{3}^{3}(-\tau)+O(4) \\
0
\end{array}\right) .
$$

Then, system (30) is equivalent to the following operator equation:

$$
\dot{u}_{t}=A(\mu) u_{t}+R(\mu) u_{t}
$$

where $u(t)=\left(x_{1}(t), y_{1}(t), x_{2}(t), y_{2}(t)\right)^{T}, u_{t}=u(t+\theta)$, for $\theta \in[-\tau, 0]$.

$$
\begin{aligned}
& \text { For } \psi \in \mathscr{C}^{1}\left([0, \tau], \mathbb{R}^{4}\right), \text { define } \\
& A^{*} \psi(s)= \begin{cases}-\frac{\mathrm{d} \psi(s)}{\mathrm{d} s}, & s \in(0, \tau], \\
\int_{-\tau}^{0} \psi(-\xi) \mathrm{d} \eta(\xi, 0), & s=0 .\end{cases}
\end{aligned}
$$

For $\phi \in \mathscr{C}[-\tau, 0]$ and $\psi \in \mathscr{C}[0, \tau]$, define the bilinear form

$$
\begin{aligned}
\langle\psi(s), \phi(\theta)\rangle= & \bar{\psi}(0) \phi(0) \\
& -\int_{-\tau}^{0} \int_{0}^{\theta} \bar{\psi}(\xi-\theta) \mathrm{d} \eta(\theta) \phi(\xi) \mathrm{d} \xi,
\end{aligned}
$$

where $\eta(\theta)=\eta(\theta, 0)$. Then, $A(0)$ and $A^{*}$ are adjoint operators.

Let $q(\theta)$ and $q^{*}(s)$ be eigenvectors of $A(0)$ and $A^{*}$ associated to $i \omega^{*}$ and $-i \omega^{*}$, respectively. It is not difficult with verify that

$$
\begin{aligned}
q(\theta) & =\left(1, \frac{\gamma_{1}}{i \omega^{*}}, 1, \frac{\gamma_{1}}{i \omega^{*}}\right)^{T} e^{i \omega^{*} \theta}, \\
q^{*}(s) & =\frac{1}{\bar{D}}\left(1, \frac{\gamma_{2}}{i \omega^{*}}, 1, \frac{\gamma_{2}}{i \omega^{*}}\right) e^{i \omega^{*} s},
\end{aligned}
$$

where

$$
D=2+\frac{2 \gamma_{1} \gamma_{2}}{\omega^{* 2}}+2 \beta^{*} \delta \gamma_{2} \tau e^{-\mathrm{i} \omega^{*} \tau}
$$

Then, $\left\langle q^{*}(s), q(\theta)\right\rangle=1,\left\langle q^{*}(s), \bar{q}(\theta)\right\rangle=0$.

Let $u_{t}$ be the solution of (39) and define

$$
z(t)=\left\langle q^{*}, u_{t}\right\rangle, \quad W(t, \theta)=u_{t}(\theta)-2 \operatorname{Re}\{z(t) q(\theta)\} .
$$

On the center manifold $\mathscr{C}_{0}$, we have

$$
W(t, \theta)=W(z(t), \bar{z}(t), \theta)
$$

where

$$
W(z, \bar{z}, \theta)=W_{20} \frac{z^{2}}{2}+W_{11} z \bar{z}+W_{02} \frac{\bar{z}^{2}}{2}+\cdots,
$$

$z$ and $\bar{z}$ are local coordinates for center manifold $\mathscr{C}_{0}$ in the direction of $q^{*}$ and $\bar{q}^{*}$. Note that $W$ is real if $u_{t}$ is real. We only consider real solutions.

For solution $u_{t}$ in $\mathscr{C}_{0}$, since $\mu=0$, we have

$$
\begin{aligned}
\dot{z}(t) & =i \omega^{*} z+\left\langle q^{*}(\theta), f(0, W+2 \operatorname{Re}\{z(t) q(\theta)\})\right\rangle \\
& =i \omega^{*} z+\bar{q}^{*}(0), f(0, W(z, \bar{z}, 0)+2 \operatorname{Re}\{z(t) q(0)\}) \\
& =i \omega^{*} z+\bar{q}^{*}(0) f_{0}(z, \bar{z})
\end{aligned}
$$

We rewrite this equation as

$$
\dot{z}(t)=i \omega^{*} z+g(z, \bar{z}),
$$

where

$$
g(z, \bar{z})=g_{20} \frac{z^{2}}{2}+g_{11} z \bar{z}+g_{02} \frac{\bar{z}^{2}}{2}+g_{21} \frac{z^{2} \bar{z}}{2} \cdots
$$

By (39) and (48), we have

$$
\begin{aligned}
\dot{W} & =\dot{u}_{t}-\dot{z} q-\dot{\bar{z}} \bar{q} \\
& = \begin{cases}A W-2 \operatorname{Re}\left\{\bar{q}^{*}(0) f_{0} q(\theta)\right\}, & \theta \in[-\tau, 0), \\
A W-2 \operatorname{Re}\left\{\bar{q}^{*}(0) f_{0} q(0)\right\}+f_{0}, & \theta=0,\end{cases} \\
& =A W+H(z, \bar{z}, \theta),
\end{aligned}
$$




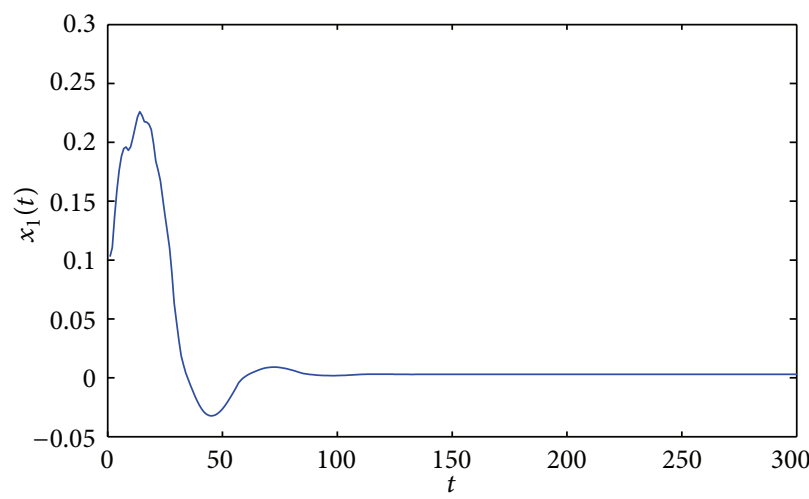

(a)

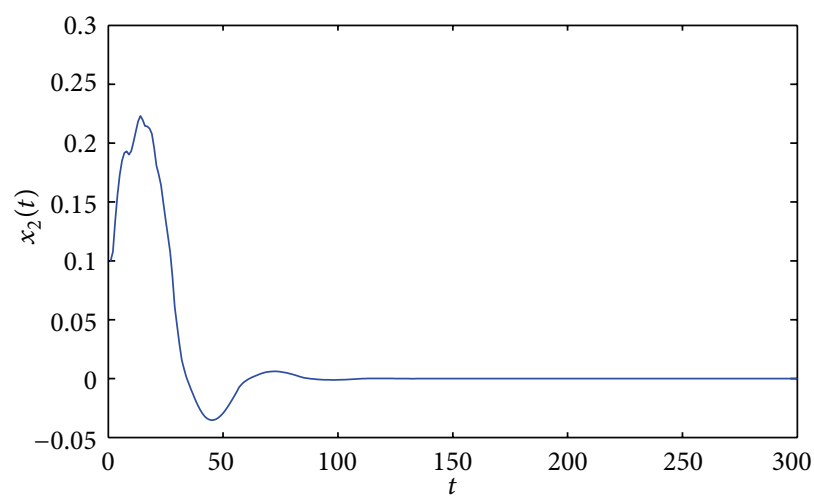

(c)

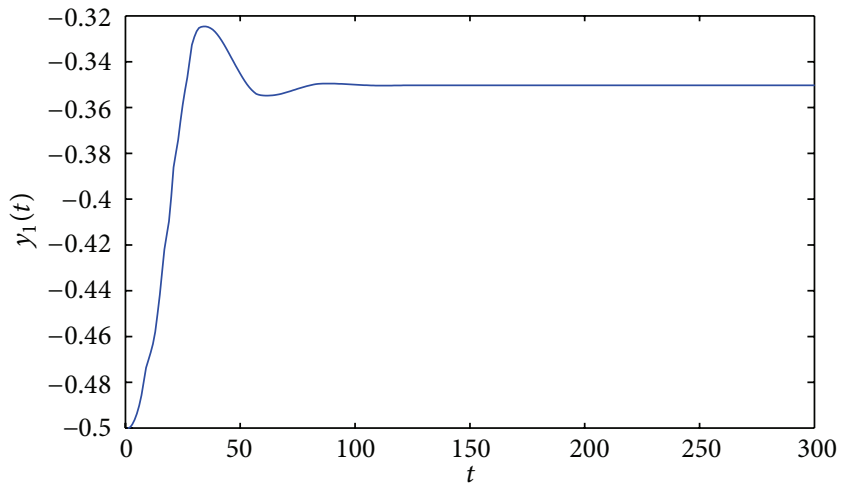

(b)

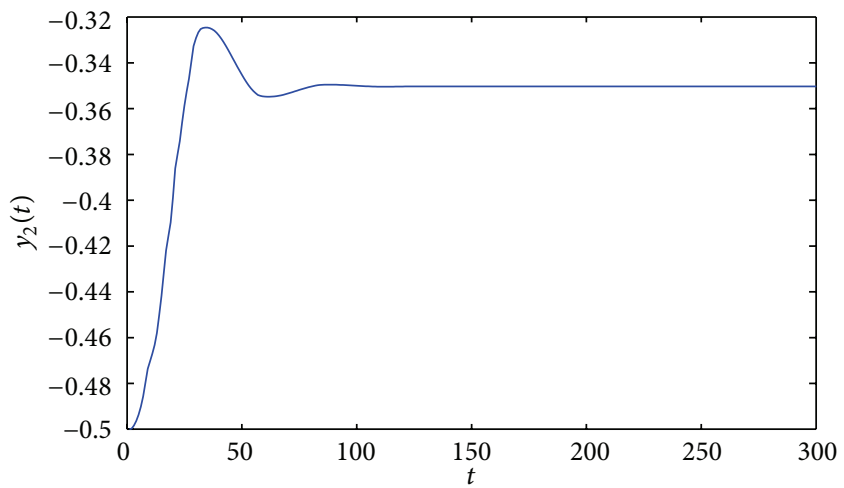

(d)

FIGURE $2: \gamma_{1}=0.1, \gamma_{2}=2.5, \tau=1.5, k=1.9$, which means that condition $\left(H_{1}\right)$ holds, and $\beta=0.7<\beta_{0}$. The initial value is $(0.1,-0.5,0.1,-0.5)$.

where

$$
H(z, \bar{z}, \theta)=H_{20}(\theta) \frac{z^{2}}{2}+H_{11}(\theta) z \bar{z}+H_{02}(\theta) \frac{\bar{z}^{2}}{2}+\cdots .
$$

Expanding the above series and comparing the coefficients, we obtain

$$
\begin{gathered}
\left(A-2 i \omega^{*} I\right) W_{20}(\theta)=-H_{20}(\theta), \\
A W_{11}(\theta)=-H_{11}(\theta), \ldots .
\end{gathered}
$$

Notice that

$$
\begin{gathered}
q(\theta)=\left(1, \frac{\gamma_{1}}{i \omega^{*}}, 1, \frac{\gamma_{1}}{i \omega^{*}}\right)^{T} e^{i \omega^{*} \theta}, \\
u_{t}(\theta)=z q(\theta)+\bar{z} \bar{q}(\theta)+W(z, \bar{z}, \theta),
\end{gathered}
$$

where

$$
\begin{aligned}
W^{(i)}(z, \bar{z}, \theta)= & W_{20}^{(i)}(\theta) \frac{z^{2}}{2}+W_{11}^{(i)}(\theta) z \bar{z} \\
& +W_{02}^{(i)}(\theta) \frac{\bar{z}^{2}}{2}+\cdots, \quad i=1,2,3,4 .
\end{aligned}
$$

Combing (38) and by straightforward computation, we can obtain the coefficients which will be used in determining the important quantities:

$$
g_{20}=\frac{2 \beta^{*} \gamma_{2} \rho}{D} e^{-2 i \omega^{*} \tau}\left(k^{2}-k+2\right) \text {, }
$$

$g_{11}=\frac{2 \beta^{*} \gamma_{2} \rho}{D}\left(k^{2}-k+2\right)$

$g_{02}=\frac{2 \beta^{*} \gamma_{2} \rho}{D} e^{2 i \omega^{*} \tau}\left(k^{2}-k+2\right)$,

$g_{21}=\frac{2 \beta^{*} \gamma_{2}}{D}\left\{\rho\left(e^{i \omega^{*} \tau} W_{20}^{(1)}(-\tau)+2 e^{-i \omega^{*} \tau} W_{11}^{(1)}(-\tau)\right)\right.$

$$
\begin{aligned}
& +k^{2} \rho\left(e^{i \omega^{*} \tau} W_{20}^{(1)}(-\tau)+2 e^{-i \omega^{*} \tau} W_{11}^{(1)}(-\tau)\right) \\
& +k(1-k) \rho \\
& \times\left(e^{-i \omega^{*} \tau} W_{11}^{(3)}(-\tau)+e^{i \omega^{*} \tau} \frac{W_{20}^{(3)}(-\tau)}{2}\right. \\
& \left.+e^{i \omega^{*} \tau} \frac{W_{20}^{(1)}(-\tau)}{2}+e^{-i \omega^{*} \tau} W_{11}^{(1)}(-\tau)\right)
\end{aligned}
$$




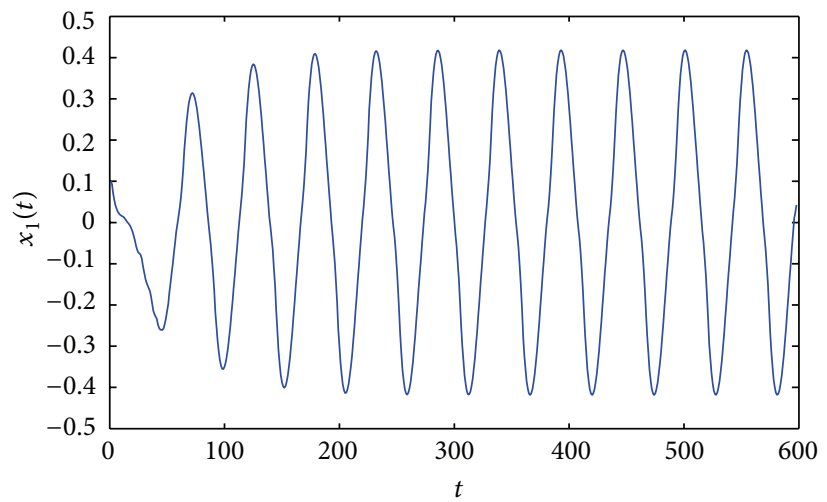

(a)

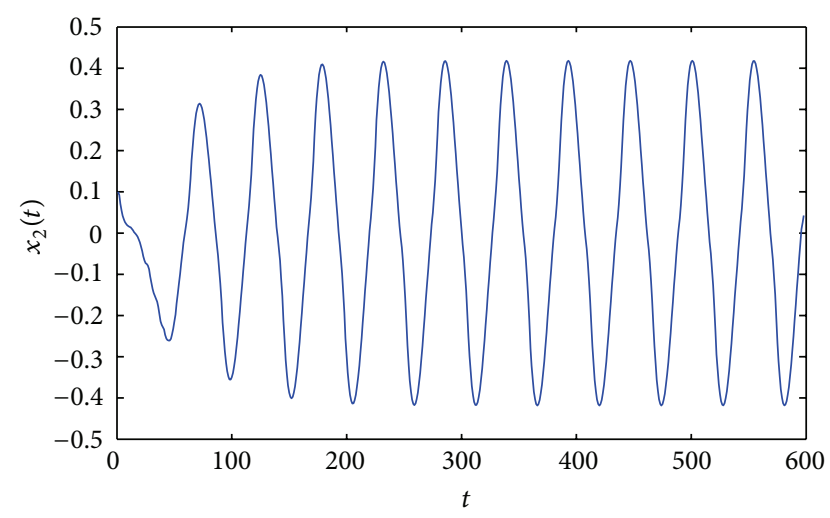

(c)

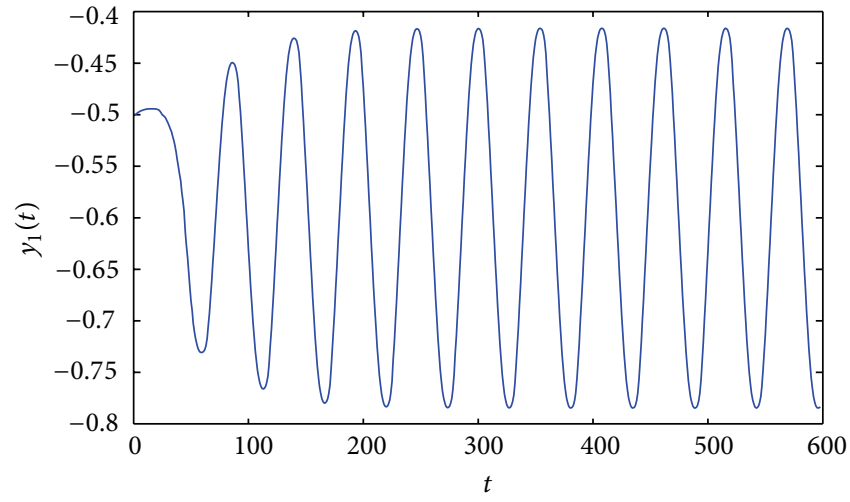

(b)

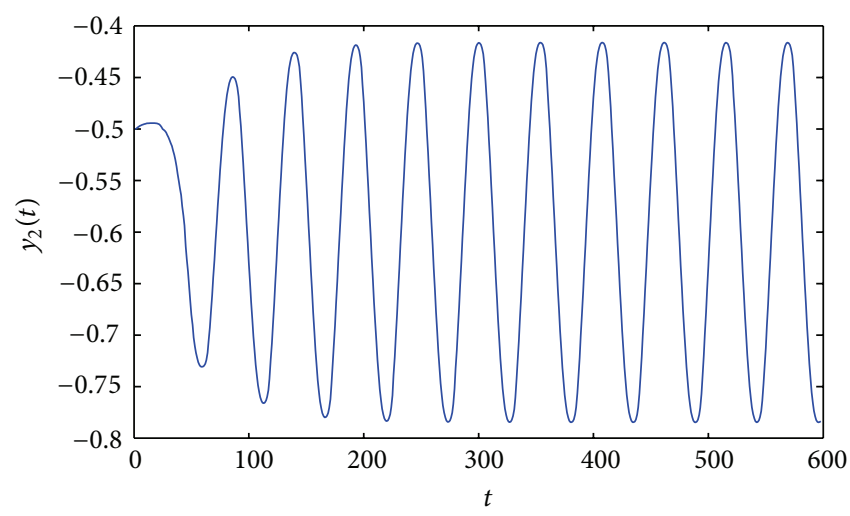

(d)

Figure 3: $\gamma_{1}=0.1, \gamma_{2}=2.5, \tau=1.5, k=1.9$, which means that condition $\left(H_{1}\right)$ holds, and $\beta=1.2>\beta_{0}$. The initial value is $(0.1,-0.5,0.1,-0.5)$.

$$
\left.\begin{array}{rl}
+(1-k)^{2} \rho\left(2 e^{-i \omega^{*} \tau} W_{11}^{(3)}(-\tau)\right. & \\
\left.+e^{i \omega^{*} \tau} W_{20}^{(3)}(-\tau)\right)-4 \delta e^{-i \omega^{*} \tau}
\end{array}\right\} .
$$

We still need to compute $W_{20}(\theta)$ and $W_{11}(\theta)$, for $\theta \in[-\tau, 0)$. We have

$$
\begin{aligned}
H(z, \bar{z}, \theta) & =-\bar{q}^{*}(0) f_{0} q(\theta)-q^{*}(0) \bar{f}_{0} \bar{q}(\theta) \\
& =-g(z, \bar{z}) q(\theta)-\bar{g}(z, \bar{z}) \bar{q}(\theta) .
\end{aligned}
$$

Comparing the coefficients about $H(z, \bar{z}, \theta)$ gives that

$$
\begin{gathered}
H_{20}(\theta)=-g_{20} q(\theta)-\bar{g}_{02} \bar{q}(\theta), \\
H_{11}=-g_{11} q(\theta)-\bar{g}_{11} \bar{q}(\theta) .
\end{gathered}
$$

Then, from (52), we get

$$
\begin{gathered}
\dot{W}_{20}(\theta)=2 i \omega^{*} W_{20}(\theta)+g_{20} q(\theta)+\bar{g}_{02} \bar{q}(\theta), \\
\dot{W}_{11}(\theta)=g_{11} q(\theta)+\bar{g}_{11} \bar{q}(\theta),
\end{gathered}
$$

which implies that

$$
\begin{gathered}
W_{20}(\theta)=\frac{g_{20} q(0)}{-i \omega^{*}} e^{i \omega^{*} \theta}+\frac{\bar{g}_{02} \bar{q}(0)}{-3 i \omega^{*}} e^{-i \omega_{j}^{*} \theta}+E e^{2 \mathrm{i} \omega_{j}^{*} \theta}, \\
W_{11}(\theta)=\frac{g_{11} q(0)}{i \omega^{*}} e^{i \omega^{*} \theta}+\frac{\bar{g}_{11} \bar{q}(0)}{-i \omega^{*}} e^{-i \omega^{*} \theta}+F .
\end{gathered}
$$

Here, $E$ and $F$ are both four-dimensional vectors and can be determined by setting $\theta=0$ in $H(z, \bar{z}, \theta)$. In fact, from (38) and

$$
H(z, \bar{z}, 0)=-2 \operatorname{Re}\left\{\bar{q}^{*}(0) f_{0} q(0)\right\}+f_{0},
$$

we have

$$
\begin{aligned}
H_{20}(0)= & -g_{20} q(0)-\bar{g}_{02} \bar{q}(0) \\
& +2 \beta^{*} \gamma_{2} \rho e^{-2 i \omega^{*} \tau}\left(1,0, k^{2}-k+1,0\right)^{T}, \\
H_{11}(0)= & -g_{11} q(0)-\bar{g}_{11} \bar{q}(0) \\
& +2 \beta^{*} \gamma_{2} \rho\left(1,0, k^{2}-k+1,0\right)^{T} .
\end{aligned}
$$

It follows from (52) and the definition of $A$ that

$$
\begin{gathered}
\beta^{*} B W_{20}(0)+\beta^{*} C W_{20}(-\tau)=2 i \omega^{*} W_{20}(0)-H_{20}(0), \\
\beta^{*} B W_{11}(0)+\beta^{*} C W_{11}(-\tau)=-H_{11}(0),
\end{gathered}
$$




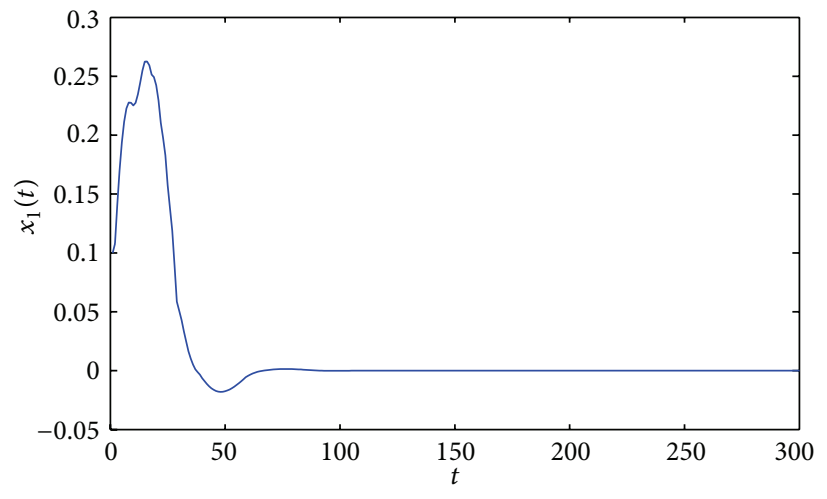

(a)

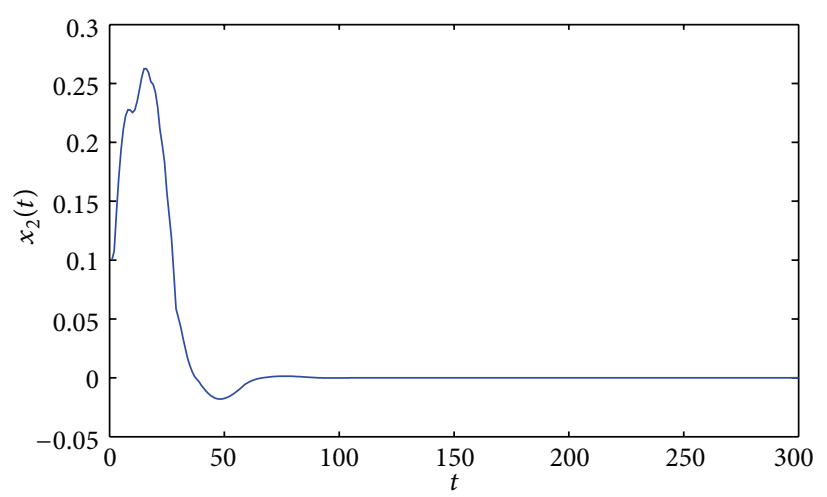

(c)

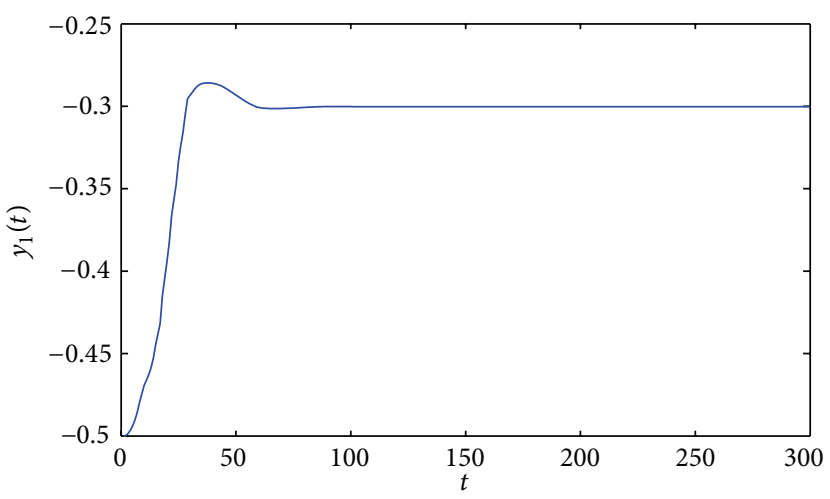

(b)

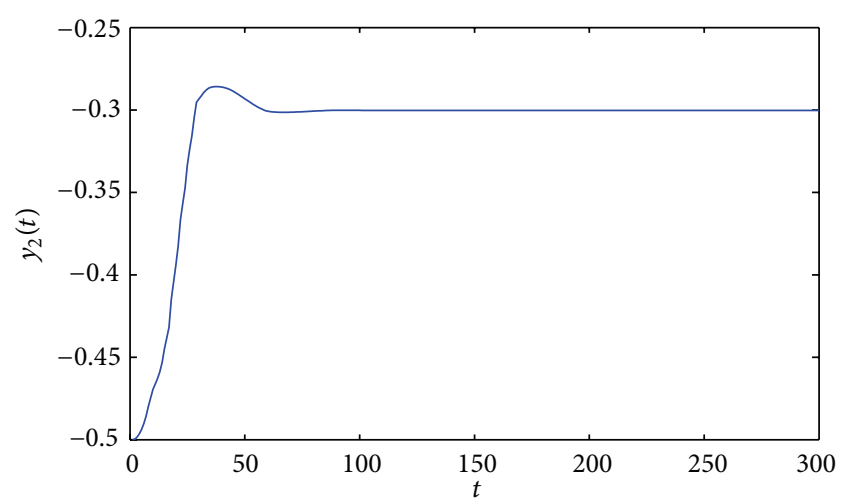

(d)

Figure 4: $\gamma_{1}=0.1, \gamma_{2}=2.5, \tau=1.5, k=3$, which means that condition $\left(H_{2}\right)$ holds, and $\beta=0.6<\bar{\beta}_{0}$. The initial value is $(0.1,-0.5,0.1,-0.5)$.

which implies that

$$
\begin{aligned}
E=(B+ & \left.e^{-2 i \omega^{*} \tau} C-2 i \omega^{*} \mathrm{I}\right)^{-1} \\
\times & {\left[\begin{array}{l}
B\left(\frac{g_{20} q(0)}{i \omega^{*}}+\frac{\bar{g}_{02} \bar{q}(0)}{3 i \omega^{*}}\right) \\
+C\left(\frac{g_{20} q(0)}{i \omega^{*}} e^{-i \omega^{*} \tau}+\frac{\bar{g}_{02} \bar{q}(0)}{3 i \omega^{*}} e^{i \omega^{*} \tau}\right) \\
+\frac{1}{\beta^{*}}\left(g_{20} q(0)+\bar{g}_{02} \bar{q}(0)\right) \\
\left.\times 2 \beta^{*} \gamma_{2} \rho e^{-2 i \omega^{*} \tau}\left(1,0, k^{2}-k+1,0\right)^{T}\right], \\
F=(B+C)^{-1}\left[B\left(\frac{g_{11} q(0)}{-i \omega^{*}}+\frac{\bar{g}_{11} \bar{q}(0)}{i \omega^{*}}\right)\right.
\end{array}\right] }
\end{aligned}
$$

$$
\begin{aligned}
& +C\left(\frac{g_{11} q(0)}{-i \omega^{*}} e^{-i \omega^{*} \tau}+\frac{\bar{g}_{11} \bar{q}(0)}{i \omega^{*}} e^{i \omega^{*} \tau}\right) \\
& +\frac{1}{\beta^{*}}\left(g_{11} q(0)+\bar{g}_{11} \bar{q}(0)\right) \\
& \left.-2 \beta^{*} \gamma_{2} \rho\left(1,0, k^{2}-k+1,0\right)^{T}\right] .
\end{aligned}
$$

Consequently, the above $g_{21}$ can be expressed by the parameters and delay in system (30). Thus, we can compute the following quantities:

$$
\begin{gathered}
c_{1}(0)=\frac{i}{2 \omega^{*}}\left(g_{20} g_{11}-2\left|g_{11}\right|^{2}-\frac{1}{3}\left|g_{20}\right|^{2}\right)+\frac{g_{21}}{2}, \\
\mu_{2}=-\frac{\operatorname{Re} c_{1}(0)}{\operatorname{Re} \lambda^{\prime}\left(\beta^{*}\right)}, \\
\beta_{2}=2 \operatorname{Re} c_{1}(0), \\
T_{2}=-\frac{\operatorname{Im} c_{1}(0)+\mu_{2} \operatorname{Im} \lambda^{\prime}\left(\beta^{*}\right)}{\omega^{*}},
\end{gathered}
$$

which determine the properties of bifurcating periodic solutions at the critical value $\tau_{0}$. The direction and stability of the 


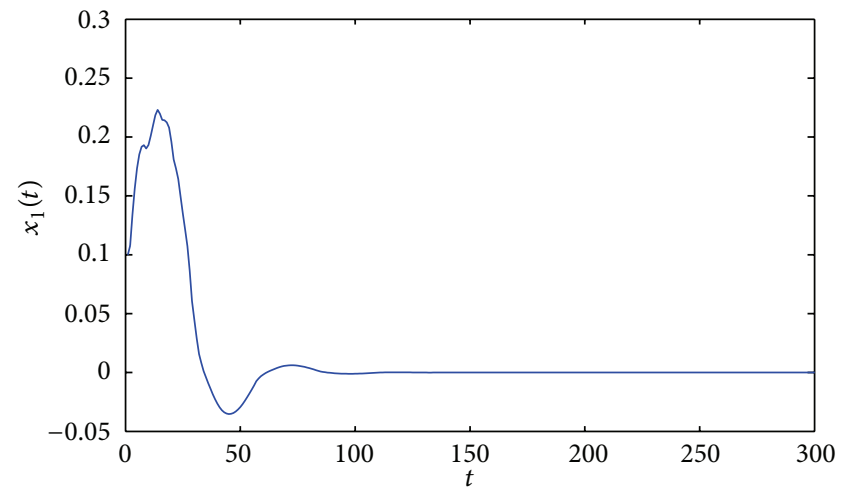

(a)

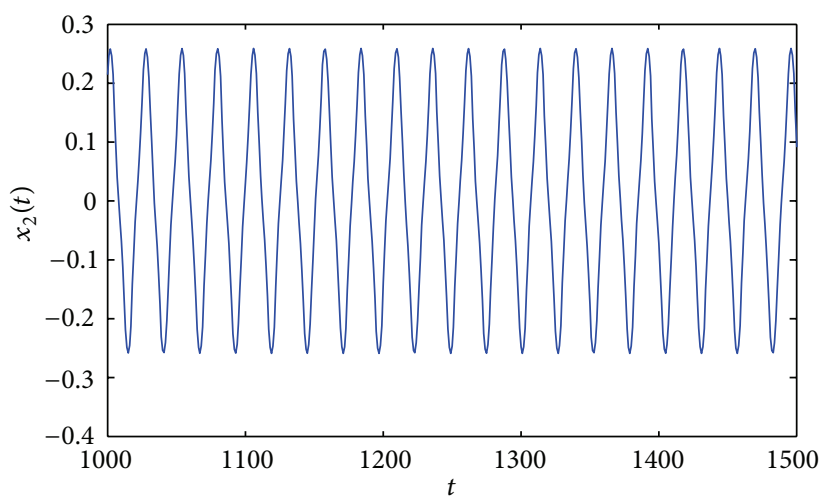

(c)

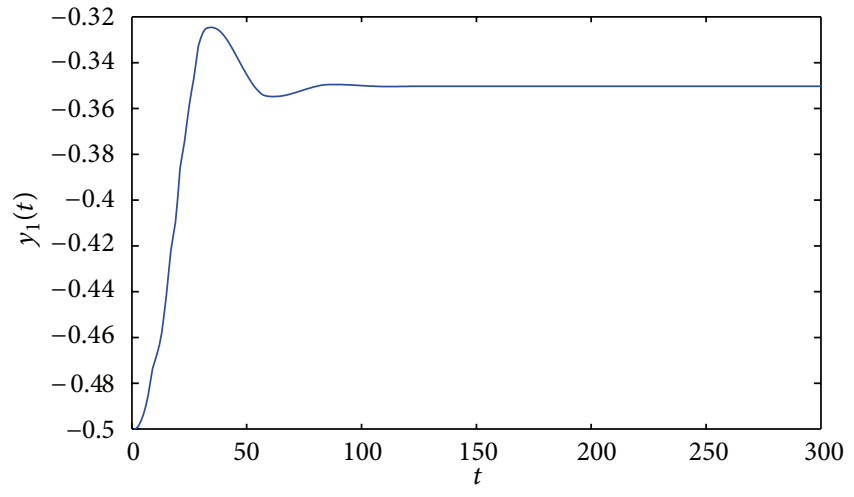

(b)

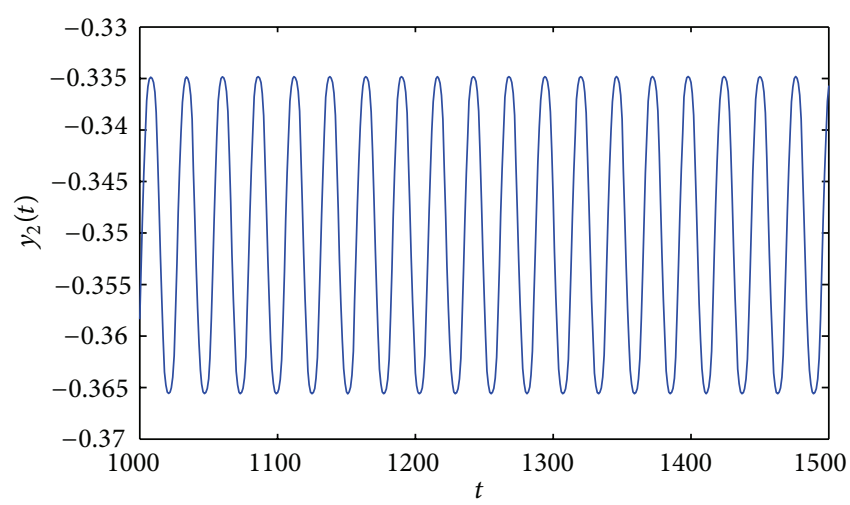

(d)

FIgURE 5: $\gamma_{1}=0.1, \gamma_{2}=2.5, \tau=1.5, k=3$, which means that condition $\left(H_{2}\right)$ holds, and $\bar{\beta}_{0}<\beta=0.7<\beta_{0}$. The initial value is $(0.1,-0.5,0.1,-0.5)$.

Hopf bifurcation in the center manifold can be determined by $\mu_{2}$ and $\beta_{2}$, respectively. In fact, if $\mu_{2}>0\left(\mu_{2}<0\right)$, then the bifurcating periodic solutions are forward (backward); the bifurcating periodic solutions on the center manifold are stable (unstable) if $\beta_{2}<0\left(\beta_{2}>0\right)$; and $T_{2}$ determines the period of the bifurcating periodic solutions: the period increases (decreases) if $T_{2}>0\left(T_{2}<0\right)$.

From the discussion in Section 2, we have known that $\operatorname{Re} \lambda^{\prime}\left(\beta_{j}\right)>0$; therefore; we have the following result.

Theorem 7. The direction of the Hopf bifurcation for system (3) at the equilibrium $E\left(0,-\beta \cos ^{2} \varphi_{0}, 0,-\beta \cos ^{2} \varphi_{0}\right)$ when $\beta=$ $\beta^{*}$ is forward (backward), and the bifurcating periodic solutions on the center manifold are stable (unstable) if $\operatorname{Re}\left(c_{1}(0)\right)<$ $0(>0)$. Particularly, the stability of the bifurcation periodic solutions of system (3) and the reduced equations on the center manifold are coincident at the first bifurcation value $\beta=\beta_{0}$.

\section{Numerical Simulations}

In this section, we will carry out numerical simulations on system (3) at special values of $\beta$. We choose a set of data as follows:

$$
\gamma_{1}=0.1, \quad \gamma_{2}=2.5, \quad \varphi_{0}=\frac{\pi}{4}, \quad \tau=1.5
$$

which are the same as those in [1]. Then, $\delta=1, \rho=0$.
Then, we can obtain

$$
\begin{gathered}
\omega_{0} \dot{=} 0.2225, \quad \omega_{1} \dot{=} 3.5677, \ldots, \\
\omega_{0} \dot{=} 1.7294, \quad \omega_{1} \dot{\doteq} 5.5531, \ldots, \\
\beta_{0} \dot{=} 1.1008, \quad \beta_{1} \dot{=} 1.7434, \ldots, \\
\bar{\beta}_{0} \dot{=} 1.3534, \quad \bar{\beta}_{1} \dot{=} 2.5235, \ldots, \quad k=1.9, \\
\bar{\beta}_{0} \dot{=} 0.6093, \quad \bar{\beta}_{1} \dot{=} 1.1356, \ldots, \quad k=3 .
\end{gathered}
$$

From the analysis in Section 2, we know that $\beta(\bar{\beta})$ is increasing with respect to $\omega(\varpi)$ when $\omega(\varpi)>\gamma_{1} \gamma_{2}$, which means that

$$
\beta_{0}=\min \left\{\beta_{j}\right\}, \quad \bar{\beta}_{0}=\min \left\{\bar{\beta}_{j}\right\}, \quad j=0,1,2, \ldots,
$$

that is, $\beta_{0}\left(\bar{\beta}_{0}\right)$ is the first critical value at which system (3) undergoes a Hopf bifurcation.

When $k=1.9$, by the previous results, it follows that

$$
\begin{gathered}
\lambda^{\prime}\left(\beta_{0}\right) \doteq 0.2440-0.0491 \mathrm{i}, \quad c_{1}(0) \stackrel{\dot{=}}{=}-0.5373+0.1082 \mathrm{i}, \\
\mu_{2} \stackrel{\doteq}{=} .2020, \quad \beta_{2} \stackrel{\doteq}{=}-1.0746, \quad T_{2}=-0.0018 .
\end{gathered}
$$




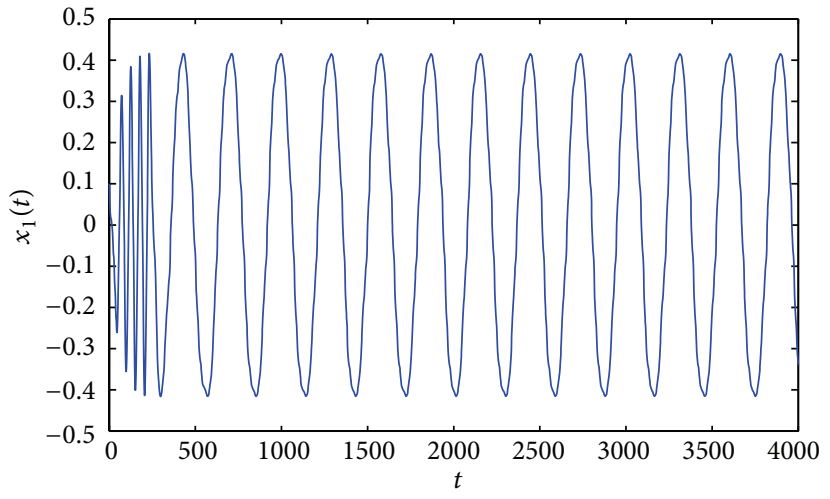

(a)

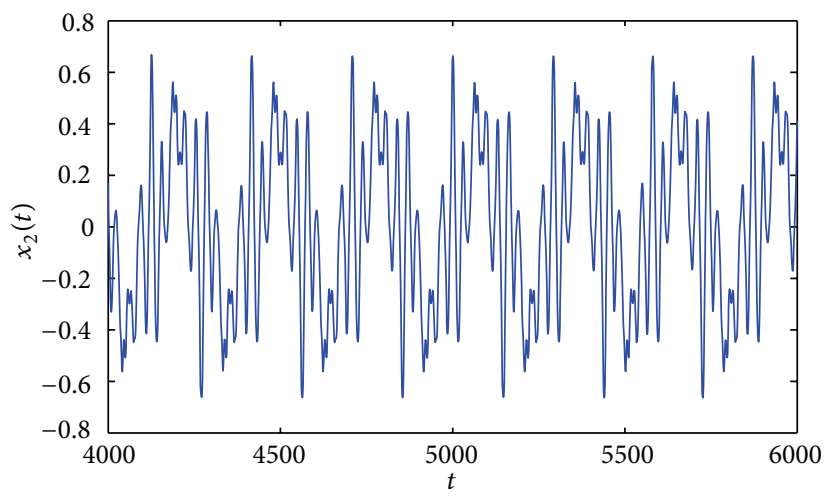

(c)

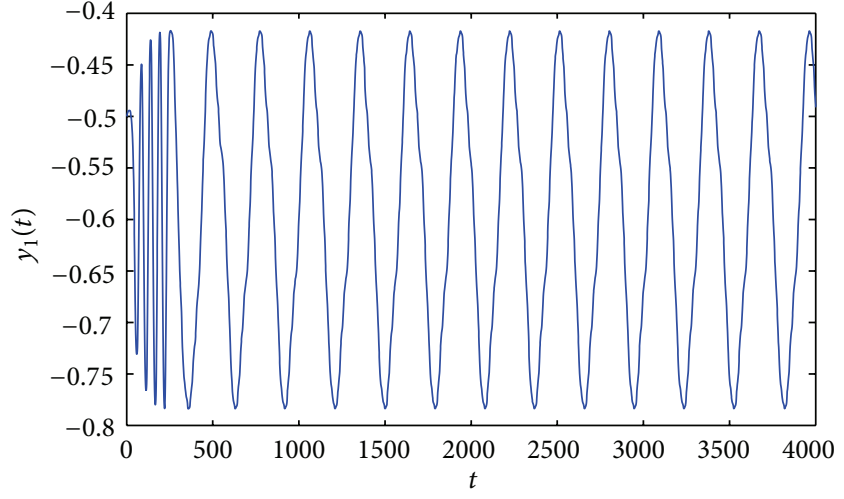

(b)

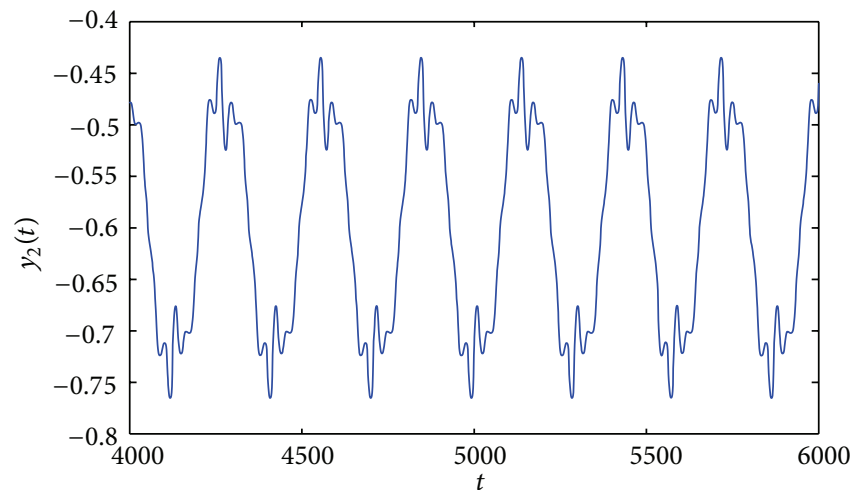

(d)

Figure 6: $\gamma_{1}=0.1, \gamma_{2}=2.5, \tau=1.5, k=3$, which means that condition $\left(H_{2}\right)$ holds, and $\beta=1.2>\beta_{0}>\bar{\beta}_{0}$. The initial value is $(0.1,-0.5,0.1,-0.5)$.

Hence, we arrive at the following conclusion: the equilibrium $E$ is asymptotically stable when $\beta \in[0,1.1008)$ and unstable when $\beta \in(1.1008,+\infty)$, and, at the first critical value, the bifurcating periodic solutions are asymptotically stable, and the direction of the bifurcation is forward (see Figures 2 and 3 ).

When $k=3$, we can get

$$
\begin{gathered}
\lambda^{\prime}\left(\bar{\beta}_{0}\right) \stackrel{\doteq}{=} 0.9004+0.0924 \mathrm{i}, \quad c_{1}(0) \stackrel{\doteq}{=}-1.9116+0.7930 \mathrm{i}, \\
\mu_{2} \stackrel{\doteq}{=} 2.1231, \quad \beta_{2} \dot{=}=-3.8232, \quad T_{2}=-0.5720 .
\end{gathered}
$$

Then, we have the following: the equilibrium $E$ is asymptotically stable when $\beta \in[0,0.6093)$, and unstable when $\beta \in(0.6093,+\infty)$, and, at the first critical value, the bifurcating periodic solutions are asymptotically stable, and the direction of the bifurcation is forward (see Figures 4, 5, and 6).

\section{Conclusion}

Ravoori et al. [1] explored an experimental system of two nominally identical optoelectronic feedback loops coupled unidirectionally, which are described by system (3). In the experiment, they found that depending on the value of the feedback strength $\beta$ and delay $\tau$, system (1) is capable of producing dynamics ranging from periodic oscillations to high-dimensional chaos $[14,15]$.

This paper investigates the stability and the existence of periodic solutions. We find that with the variety of the coupling strength $k$, even if all other parameters keep the same, the dynamical behavior can change greatly. In fact, it is clear that the first two equations, $x_{1}(t)$ and $y_{1}(t)$ are uncoupled with equations $x_{2}(t)$ and $y_{2}(t)$, so system (1) are independent of (2), which means that coupling strength $k$ does not appear in (1). The characteristic equation of (1) has the same form as (6), so the first critical value $\beta_{0}$ is independent of $k$. The analysis of characteristic equation (7) shows that the value of $k$ can affect the first critical value $\bar{\beta}_{0}$ definitely. And we draw a conclusion that when $k$ is in an interval, in which $\beta_{0}<\bar{\beta}_{0}$ holds, solutions of system (1) and (2) keep synchronous; when $k$ belongs to the interval, in which $\bar{\beta}_{0}<\beta_{0}$ holds, solutions of system (1) and (2) can also keep synchronous with $\beta<\bar{\beta}_{0}$, while they lose their synchronization when $\beta>\bar{\beta}_{0}$, no matter whether $\beta<\beta_{0}$ or not.

As a result, the modulation of the coupling strengths $k$ together with the feedback strength $\beta$ would be an efficient and an easily implementable method to control the behavior of the coupled chaotic oscillators. 


\section{Acknowledgment}

This paper is supported by the National Natural Science Foundation of China (no. 11031002) and the Research Fund for the Doctoral Program of Higher Education of China (no. 20122302110044).

\section{References}

[1] B. Ravoori, A. B. Cohen, A. V. Setty et al., "Adaptive synchronization of coupled chaotic oscillators," Physical Review E, vol. 80, no. 5, Article ID 056205, 2009.

[2] L. M. Pecora and T. L. Carroll, "Master stability functions for synchronized coupled systems," Physical Review Letters, vol. 80, no. 10, pp. 2109-2112, 1998.

[3] K. M. Cuomo and A. V. Oppenhein, "Circuit implementation of synchronized chaos with applications to communications," Physical Review Letters, vol. 71, no. 1, pp. 65-68, 1993.

[4] J. P. Goedgebuer, L. Larger, and H. Porte, “Optical cryptosystem based on synchronization of hyperchaos generated by a delayed feedback tunable laser diode," Physical Review Letters, vol. 80, no. 10, pp. 2249-2252, 1998.

[5] A. Arenas, A. DÃaz-Guilera, J. Kurths, Y. Moreno, and C. Zhou, "Synchronization in complex networks," Physics Reports, vol. 469, no. 3, pp. 93-153, 2008.

[6] T. Heil, I. Fischer, W. Elsäßer, and A. Gavrielides, "Dynamics of semiconductor lasers subject to delayed optical feedback: the short cavity regime," Physical Review Letters, vol. 87, no. 24, Article ID 243901, 4 pages, 2001.

[7] T. Heil, I. Fischer, W. Elsäßer, J. Mulet, and C. R. Mirasso, "Chaos synchronization and spontaneous symmetry-breaking in symmetrically delay-coupled semiconductor lasers," Physical Review Letters, vol. 86, no. 5, pp. 795-798, 2001.

[8] D. V. Ramana Reddy, A. Sen, and G. L. Johnston, "Time delay induced death in coupled limit cycle oscillators," Physical Review Letters, vol. 80, no. 23, pp. 5109-5112, 1998.

[9] R. Lang and K. Kobayashi, "External optical feedback effects on semiconductor injection laser properties," IEEE Journal of Quantum Electronics, vol. 16, no. 3, pp. 347-355, 1980.

[10] P. M. Alsing, V. Kovanis, A. Gavrielides, and T. Erneux, "Lang and Kobayashi phase equation," Physical Review A, vol. 53, no. 6, pp. 4429-4434, 1996.

[11] M. M. Möhrle, B. Sartorius, C. Bornholdt et al., "Detuned grating multisection-RW-DFB lasers for high-speed optical signal processing," IEEE Journal of Selected Topics in Quantum Electronics, vol. 7, no. 2, pp. 217-223, 2001.

[12] J. Wei and C. Yu, "Stability and bifurcation analysis in the crosscoupled laser model with delay," Nonlinear Dynamics, vol. 66, no. 1-2, pp. 29-38, 2011.

[13] I. Fischer, Y. Liu, and P. Davis, "Synchronization of chaotic semiconductor laser dynamics on subnanosecond time scales and its potential for chaos communication," Physical Review A, vol. 62, no. 1, Article ID 011801, 4 pages, 2000.

[14] A. B. Cohen, B. Ravoori, T. E. Mruphy, and R. Roy, "Using synchronization for prediction of high-dimensional chaotic dynamics," Physical Review Letters, vol. 101, no. 15, Article ID 154102, 2008.

[15] Y. C. Kouomou, P. Colet, L. Larger, and N. Gastaud, "Chaotic breathers in delayed electro-optical systems," Physical Review Letters, vol. 95, no. 20, Article ID 203903, 4 pages, 2005.
[16] S. Ruan and J. Wei, "On the zeros of transcendental functions with applications to stability of delay differential equations with two delays," Dynamics of Continuous, Discrete \& Impulsive Systems. Series A, vol. 10, no. 6, pp. 863-874, 2003.

[17] B. D. Hassard, N. D. Kazarinoff, and Y. H. Wan, Theory and Applications of Hopf Bifurcation, vol. 41 of London Mathematical Society Lecture Note Series, Cambridge University Press, Cambridge, UK, 1981.

[18] J. Hale, Theory of Functional Differential Equations, vol. 3, Springer, New York, NY, USA, 2nd edition, 1977, Applied Mathematical Sciences. 


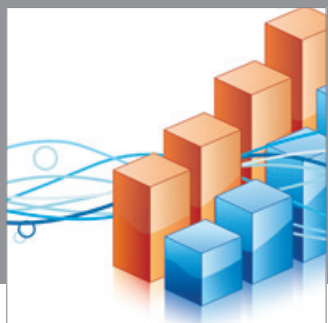

Advances in

Operations Research

mansans

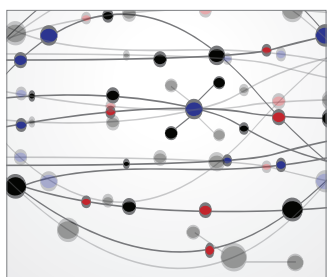

The Scientific World Journal
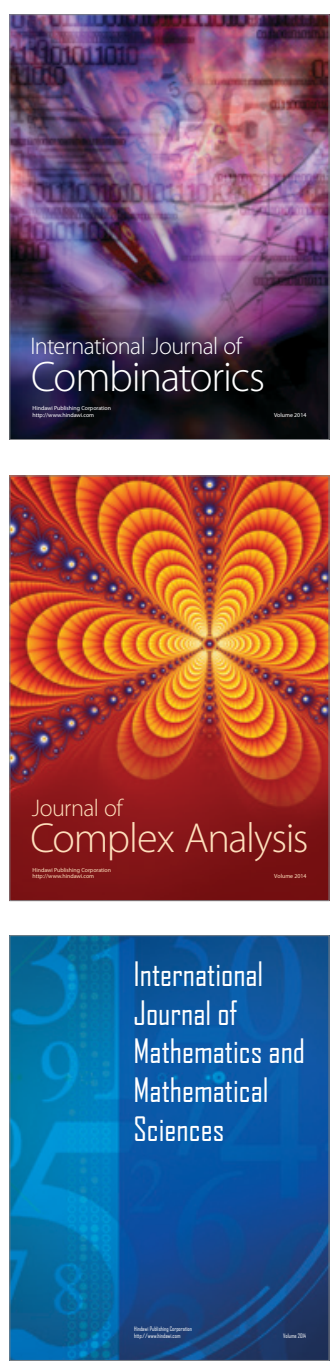
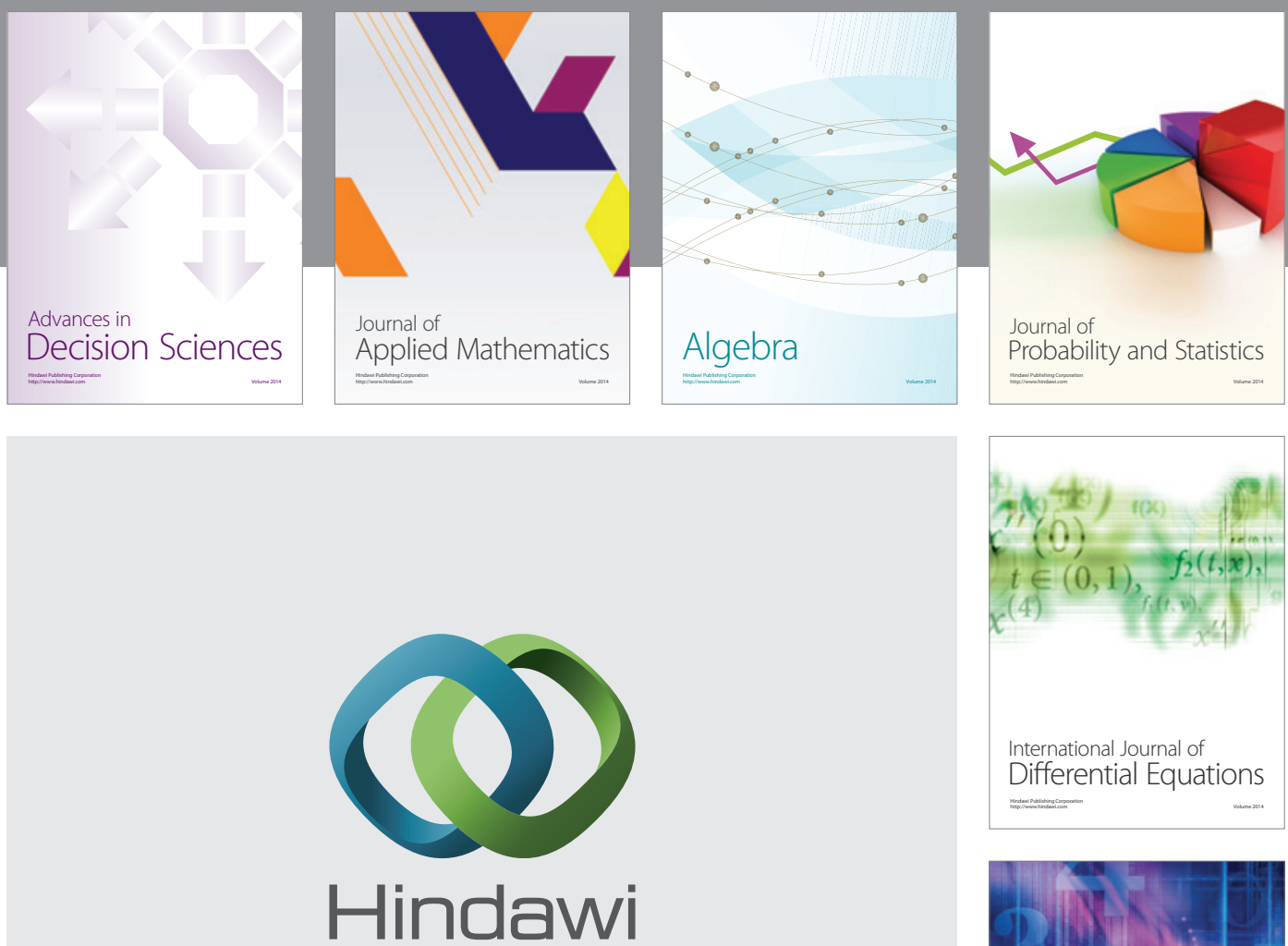

Submit your manuscripts at http://www.hindawi.com
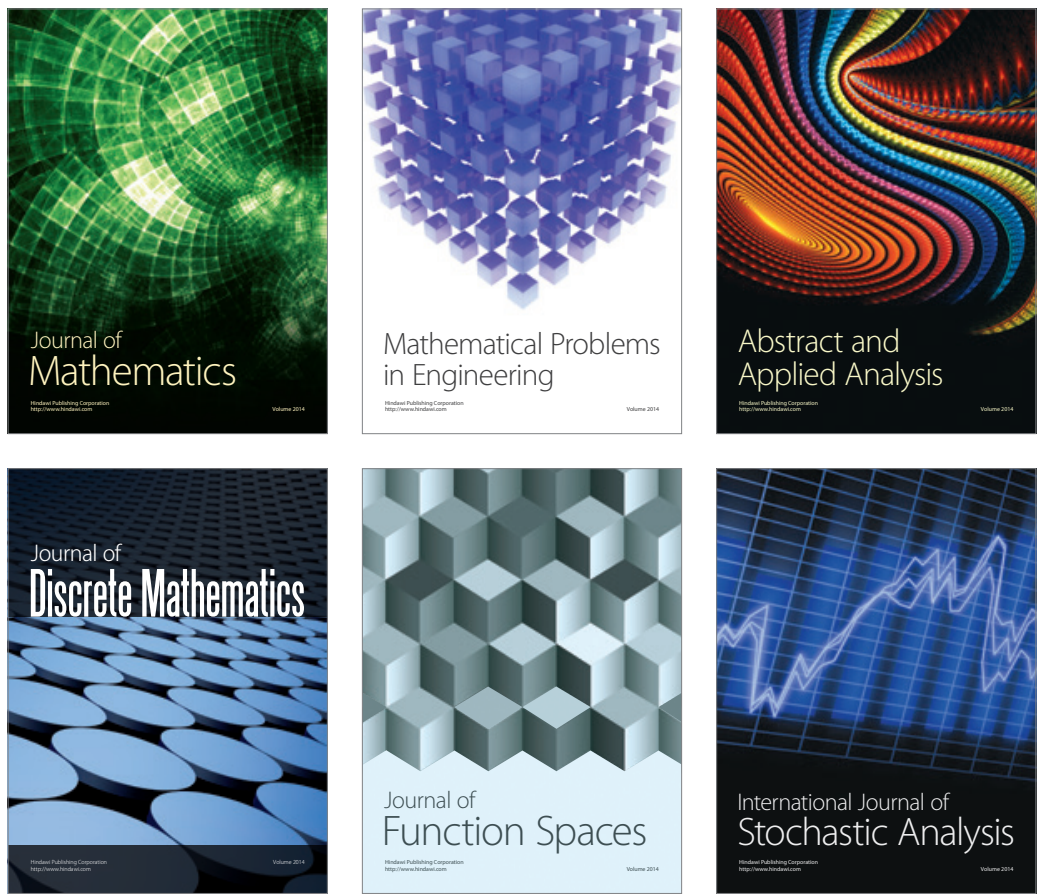

Journal of

Function Spaces

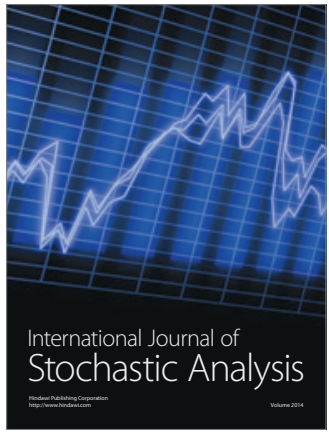

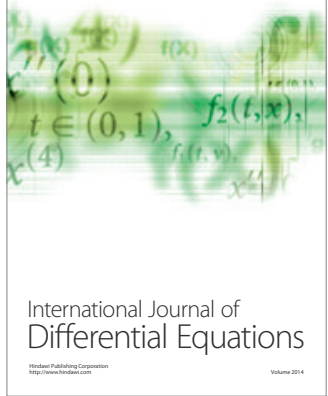
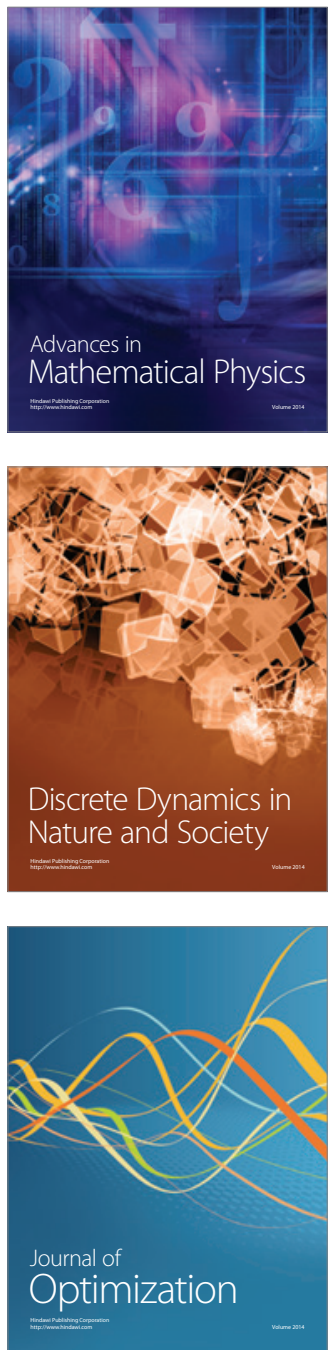